\title{
Research on Modeling and Key Technologies of Aerobics Intelligent Teaching System
}

\author{
Xiaoxuan Huang, Jun Zong \\ Jiangxi Science \& Technology Normal University, Jiangxi Province, Nanchang, 330013
}

Keywords: aerobics; intelligent teaching system; key technology

\begin{abstract}
With the continuous development of the information age, the amount of knowledge has also increased, and its speed of communication has become faster and faster. The modern education field must also accelerate the development and construction of new educational models, so as to keep up with the development needs of the times. The society has cultivated more outstanding talents to ensure the long-term development of the education industry. Taking aerobics teaching as an example, this paper discusses the modeling and key technologies of its intelligent teaching system, which can be used by peers.
\end{abstract}

\section{Introduction}

In the new era of rapid development of artificial intelligence technology, artificial intelligence has also been gradually applied to the field of education, and has formed an intelligent teaching system. The intelligent teaching system is a comprehensive system involving many disciplines and fields, such as psychology, computer science, behavioral science, artificial intelligence, cognitive science, and education. The aerobics intelligent teaching system can help students to learn aerobics by integrating intelligence into the computer system, and even replace the aerobics teacher to some extent, and its teaching effect is ideal. However, there are few studies on the aerobics intelligent teaching system in China, which deserves our in-depth discussion.

\section{Overview of Intelligent Teaching System}

The intelligent teaching system integrates the teaching and research results of various senior and special-level teaching experts and teachers across the country with teaching resources by means of Internet computer artificial simulation technology, and analyzes and organizes them. Based on the results of intelligent judgment, it is targeted, effective and real-time [1]. The intelligent teaching system can automatically find out the blind spots that teachers have in teaching and students' learning, and automatically process them. This will help reduce the learning pressure of students and enhance the teaching effect, thus cultivating more for social development. The intelligent teaching system has experienced three stages of development: 1 The first generation teaching system, that is, the school examination system, is a learning and diagnosis method. Due to its limited teaching experience, narrow knowledge and long interval of diagnosis, students cannot be real-time. Mastering the effectiveness of learning, it is gradually eliminated; 2 The second generation of teaching system, namely the personality learning system, although this method can accurately identify the weak points of students' learning, however, because of the limited location and geographical area, it is impossible to maximize Limiting the efficiency of learning; 3 The third-generation teaching system, that is, the intelligent teaching system, can realize the blind spots of learning in real time and accurately, reduce the pressure on students, and maximize the learning effect of students. It is an ideal teaching at present.

\section{The Model Construction of Aerobics Intelligent Teaching System}

The expert knowledge module has the following functions: First, it is the hub of other modules that frequently use the system, can realize real-time response to user behavior, utilize knowledge 
base knowledge, propose corresponding tasks and problems, and explain; secondly, it can Synchronous answers to student questions, and by comparing students' actual behavior with expected behaviors, can predict students' learning style, learning status and knowledge of knowledge.

An important technology of expert knowledge module research is knowledge representation, which is directly related to the success or failure of aerobics intelligent teaching system. The means of knowledge representation mainly include the following: First, the production rule, which belongs to a more effective and simple knowledge representation tool, can simultaneously express procedural knowledge and definitional knowledge in a unified manner. The second is a logical representation, each of which can be used to manipulate the content it represents. The third is the semantic network and framework, which is used to describe the defining knowledge of aerobics and the semantic association between various aerobic physical entities [2].

A quality student model should fully reflect the student's learning, knowledge and cognitive skills. By analyzing expert behavior and student behavior, the students' intelligent personality simulation model is established based on the personality characteristics, cognitive ability and knowledge mastery. There are three main types, namely, cognitive student model, difference model and coverage model [3].

The construction of the student model must be based on mastering the aerobics teaching curriculum. First of all, the aerobics course must be designed, that is, the adaptive aerobics teaching curriculum should be adapted to the intelligent teaching system model design to enhance the teaching effect. Secondly, the aerobics course should be selected reasonably, that is, some aerobics teaching courses should be selected as a template. And will be entered into the intelligent teaching system.

The teacher model is based on the principle of aerobics teaching, select appropriate teaching content, and then at a certain moment, in a certain expression, use the interface to present to the students, and guide the teacher how to do aerobics teaching. Aerobics teaching methods are the key to determining the effectiveness of teaching. It is recommended to adopt a variety of ways to demonstrate aerobics expertise. Because students' initial knowledge of aerobics professional knowledge can't reach the level of experts, students' perception of things will change in the process of reaching experts' knowledge level. The system must solve the problem of changing students' knowledge in time. . Therefore, a variety of teacher models should be designed according to the level of aerobics of different students.

The intelligent human-machine interface is a hub for realizing user interaction with the system, which can help other modules to acquire user behavior and information, intelligent input multimedia knowledge, and intelligent output knowledge. The intelligent human-machine interface is mainly responsible for maintaining the knowledge base, handling natural language and human-machine dialogue, and adjusting the teacher model and student model.

The construction of intelligent human-machine interface can fully reflect the essential characteristics of aerobics "learning" and "teaching". It is also the basis for establishing a formal model. The established formal model can reason and perceive through new perceived things. Learning, giving the teaching system dynamic resilience, and effectively solving the interaction between the user and the system.

\section{The Key Technology of Aerobics Intelligent Teaching System}

From a technical point of view, intelligent agent technology involves many technologies and integrates many practical application features, which helps to expand the use function of the aerobics intelligent teaching system and realize the intelligent processing of the user's commissioned tasks. Intelligent agent technology can help students better search for effective knowledge, improve information search efficiency, and encourage students to learn and interact with students. The use of intelligent agent technology in the aerobics intelligent teaching system is mainly reflected in the following aspects: First, humanized interaction and teaching mode design. The use of intelligent agent technology in the aerobics intelligent teaching system can 
fundamentally change the student's interaction mode and teaching system, so that students can experience a completely different teaching atmosphere, and the computer can adjust the teaching process in time for the students' problems. The second is collaborative learning and intelligent design. In the past, computer network-assisted instruction, students can only communicate statically. The application of intelligent agent technology in the teaching system can realize real-time analysis and sorting of the discussion panel problems. At the same time, it can also design targeted discussions based on the discussion, and lead students to conduct in-depth discussions and realize collaborative and interactive learning. The third is the self-evolution function design. Because the intelligent agent technology has the evolutionary function, it can create a dedicated intelligent agent for all students. In the process of interacting with students, it can deepen the understanding of students and lay a foundation for establishing a teaching system more suitable for aerobics students. The fourth is the open learning model design. The aerobics intelligent learning system under intelligent agent technology can provide students with an open learning atmosphere. The system covers many resources. Students can choose their own learning methods and determine their own learning priorities. This will not only help students better solve various problems. Learning problems and helping to improve students' ability to deal with problems.

Machine learning technology is an indispensable technology in all intelligent teaching systems. For example, machine learning techniques can analyze the student's problem-solving preferences based on the student's answers to the questions. Data mining technology, statistical learning technology, neural network technology and inductive logic programming are all common machine learning techniques, and are widely used in intelligent teaching [4].

The natural language intelligent processing technology is mainly applied to the construction of the intelligent human-machine interface of the aerobics intelligent teaching system, which can significantly improve the intelligent level of the system. For example, the application of intelligent fuzzy query technology helps to find knowledge from the expert knowledge base and improve the efficiency of knowledge use. The application of semantic network technology helps to reflect the semantic association and hierarchical relationship between different knowledge points. The establishment of a language human-machine interface helps to simplify the human-computer interaction process.

\section{Conclusion}

All in all, the design and application of the aerobics intelligent teaching system can better guide students to learn aerobics help to reduce the stress of aerobics teachers, and let them focus on coordinating students and aerobics intelligent teaching systems, students the relationship with the students truly plays its leading role. China's aerobics intelligent teaching system is in the early stage of development and has a broad space for development, which deserves our in-depth study.

\section{References}

[1] Li Juan. Inheritance and Transcendence-On the Effectiveness of New Approaches to Aerobics Teaching [J]. Sports technology, 2017, 38(05):158+160.

[2] Wang Yuzhen.Application Analysis of Multimedia Technology in College Aerobics Teaching [J].Voice of the Yellow River, 2017(18):123-124.

[3] Lu Li. Design and research of aerobics virtual teaching system based on information technology [J]. Electronic Design Engineering, 2017 (16): 146-149.

[4] Tang Yangcheng. Construction and demonstration of the teaching evaluation model of "multidimensional system" in aerobics class [J]. Contemporary Sports Science and Technology, 2017 (11): 248+250. 